PROCEEDINGS OF THE

AMERICAN MATHEMATICAL SOCIETY

Volume 134, Number 3, Pages 707-714

S 0002-9939(05)08180-3

Article electronically published on September 28, 2005

\title{
POSITIVITY AND STRONG ELLIPTICITY
}

\author{
A. F. M. TER ELST, DEREK W. ROBINSON, AND YUEPING ZHU
}

(Communicated by Andreas Seeger)

\begin{abstract}
We consider partial differential operators $H=-\operatorname{div}(C \nabla)$ in divergence form on $\mathbf{R}^{d}$ with a positive-semidefinite, symmetric, matrix $C$ of real $L_{\infty}$-coefficients, and establish that $H$ is strongly elliptic if and only if the associated semigroup kernel satisfies local lower bounds, or, if and only if the kernel satisfies Gaussian upper and lower bounds.
\end{abstract}

The classical Nash-De Giorgi [Nas, [DeG] theory analyzes positive second-order partial differential operators in divergence form, i.e., operators

$$
H=-\sum_{i, j=1}^{d} \partial_{i} c_{i j} \partial_{j},
$$

where $\partial_{i}=\partial / \partial x_{i}$, the coefficients $c_{i j}$ are real $L_{\infty}$-functions and the matrix $C=$ $\left(c_{i j}\right)$ is assumed to be symmetric and positive-definite almost everywhere. The starting point of the theory is the strong ellipticity assumption,

$$
C \geq \mu I>0
$$

almost everywhere, and the principal conclusion is the local Hölder continuity of weak solutions of the associated elliptic and parabolic equations. In Nash's approach the Hölder continuity of the elliptic solution is derived as a corollary of continuity of the parabolic solution, and the latter is established by an iterative argument from good upper and lower bounds on the fundamental solution. Aronson Aro subsequently improved Nash's bounds and proved that the fundamental solution of the parabolic equation, the heat kernel, satisfies Gaussian upper and lower bounds. Specifically the kernel $K$ of the semigroup $S$ is a symmetric function over $\mathbf{R}^{d} \times \mathbf{R}^{d}$ satisfying bounds

$$
a^{\prime} G_{b^{\prime} ; t}(x-y) \leq K_{t}(x ; y) \leq a G_{b ; t}(x-y)
$$

for some $a, a^{\prime}, b, b^{\prime}>0$, uniformly for all $x, y \in \mathbf{R}^{d}$ and $t>0$, where $G_{b ; t}(x)=$ $t^{-d / 2} e^{-b|x|^{2} t^{-1}}$. Background information and references can be found in the books and reviews [Dav, DER, Gri], Str1, Str2].

Received by the editors September 30, 2004.

2000 Mathematics Subject Classification. Primary 35Jxx.

Key words and phrases. Elliptic operator, semigroup, kernel, upper bounds, lower bounds.

This work was carried out while the first author was visiting the Centre for Mathematics and its Applications at the Australian National University. He thanks the Australian Research Council for its support and the CMA for its hospitality. The third author was an ARC Research Associate for the duration of the collaboration.

(C)2005 American Mathematical Society 
In this note we observe that a converse statement is true. If $H$ is an elliptic operator of the form (11), then the corresponding heat kernel satisfies the Aronson bounds (3) if and only if $H$ satisfies the strong ellipticity condition (2). In fact we show that (2) and (3) are both equivalent to lower bounds $K_{t}(x ; y) \geq a t^{-d / 2}$ for all $|x-y| \leq r t^{1 / 2}$ and $t \in\langle 0,1]$.

In the Nash-De Giorgi theory the strong ellipticity assumption (2) is first used to give a precise definition of $H$ as a positive self-adjoint operator on the complex Hilbert space $L_{2}\left(\mathbf{R}^{d}\right)$ through quadratic form techniques. Specifically, one defines the quadratic form $h$ on $L_{2}\left(\mathbf{R}^{d}\right)$ by

$$
h(\varphi)=\sum_{i, j=1}^{d} \int_{\mathbf{R}^{d}} d x \overline{\left(\partial_{i} \varphi\right)(x)} c_{i j}(x)\left(\partial_{j} \varphi\right)(x)
$$

with domain $D(h)=W^{1,2}\left(\mathbf{R}^{d}\right)=\bigcap_{i=1}^{d} D\left(\partial_{i}\right)=D\left(\Delta^{1 / 2}\right)$, where $\Delta$ denotes the selfadjoint Laplacian, i.e., $\Delta=-\sum_{i=1}^{d} \partial_{i}^{2}$, on $L_{2}\left(\mathbf{R}^{d}\right)$. Then $h$ is positive, symmetric, densely-defined and as a direct consequence of (2) it is also closed. Therefore there is a unique, positive, self-adjoint operator $H$, with $D(H) \subset D(h)$, canonically associated with $h$. In particular $(\varphi, H \varphi)=h(\varphi)$ for all $\varphi \in D(H)$.

Since our intention is to analyze the operator $H$ without the strong ellipticity assumption (2), the foregoing definition of $H$ is not applicable, and one has to adopt an alternative approach. One can still introduce the form $h$ as above, but there is no reason for the form to be closable. (For examples of non-closable $h$ see $[\mathrm{FOT}$, Theorem 3.1.6.) We circumvent this problem by a 'viscosity' method.

Let $l$ be the closed quadratic form associated with the Laplacian $\Delta$, i.e.,

$$
l(\varphi)=\sum_{i=1}^{d}\left\|\partial_{i} \varphi\right\|_{2}^{2}=\left\|\Delta^{1 / 2} \varphi\right\|_{2}^{2}
$$

with $D(l)=D\left(\Delta^{1 / 2}\right)$. Then for each $\varepsilon \in\langle 0,1]$ define $h_{\varepsilon}$ by $D\left(h_{\varepsilon}\right)=D(h)=D(l)$ and

$$
h_{\varepsilon}(\varphi)=h(\varphi)+\varepsilon l(\varphi),
$$

where $h$ denotes the form given by (4). Since $h$ is positive, the form $h_{\varepsilon}$ satisfies the strong ellipticity condition

$$
h_{\varepsilon}(\varphi) \geq \varepsilon l(\varphi)
$$

for all $\varphi \in D(h)$. In addition it satisfies the upper bounds

$$
h_{\varepsilon}(\varphi) \leq(1+\|C\|) l(\varphi),
$$

where $\|C\|$ is the essential supremum of the matrix norm of $C(x)=\left(c_{i j}(x)\right)$. It follows immediately from (66) and (7) that $h_{\varepsilon}$ is closed. Therefore there is a positive self-adjoint operator $H_{\varepsilon}$ canonically associated with $h_{\varepsilon}$. The operator $H_{\varepsilon}$ is the strongly elliptic operator with coefficients $C+\varepsilon I$. But $\varepsilon \mapsto h_{\varepsilon}(\varphi)$ decreases monotonically as $\varepsilon$ decreases for each $\varphi \in D(h)$. Therefore it follows from a result of Kato, Kat Theorem VIII.3.11, that the $H_{\varepsilon}$ converge in the strong resolvent sense, as $\varepsilon \rightarrow 0$, to a positive self-adjoint operator $H_{0}$, which we will refer to as the viscosity operator with coefficients $C=\left(c_{i j}\right)$. The strong resolvent convergence also implies that the positive contractive semigroups $S^{(\varepsilon)}$ generated by the $H_{\varepsilon}$ converge strongly to the semigroup $S^{(0)}$ generated by $H_{0}$. Therefore $S^{(0)}$ is positive and contractive on $L_{2}\left(\mathbf{R}^{d}\right)$. 
Let $h_{0}$ denote the form associated with $H_{0}$, i.e., $D\left(h_{0}\right)=D\left(H_{0}^{1 / 2}\right)$ and $h_{0}(\varphi)=$ $\left\|H_{0}^{1 / 2} \varphi\right\|_{2}^{2}$. There is an alternative method of defining $h_{0}$ which shows that it has more universal significance.

One may associate with any positive quadratic form $h$ a unique maximal closable minorant $h_{r}$, i.e., $h_{r}$ is the largest closable positive quadratic form which is majorized by $h$ (see [Sim2, Dal]). Then $h_{0}$ is the closure of $h_{r}$. In particular, if $h$ is closable, then $h_{0}$ is its closure. In addition, $h_{0}$ is the largest closed positive quadratic form which is majorized by $h$. Consequently $D(h) \subseteq D\left(h_{0}\right)$. One may characterize $D\left(h_{0}\right)$ as the vector space of all $\varphi \in L_{2}$ for which there are $\varphi_{1}, \varphi_{2}, \ldots \in D(h)$ such that $\lim _{n \rightarrow \infty} \varphi_{n}=\varphi$ in $L_{2}$ and $\liminf _{n \rightarrow \infty} h\left(\varphi_{n}\right)<\infty$. Moreover, $h_{0}(\varphi)$ equals the minimum of all $\lim _{\inf _{n \rightarrow \infty}} h\left(\varphi_{n}\right)$, where the minimum is taken over all $\varphi_{1}, \varphi_{2}, \ldots \in D(h)$ such that $\lim _{n \rightarrow \infty} \varphi_{n}=\varphi$ in $L_{2}$. (See [Sim1, Theorem 3.) In convergence theory $h_{0}$ is variously called the lower semi-continuous regularization of $h$ EkT], page 10, or the relaxed form [Dal], page 28.

The following theorem gives a precise formulation of the characterizations of strong ellipticity mentioned above. Other characterizations are given in Proposition 2

Theorem 1. Let $H_{0}$ be the viscosity operator with coefficients $C=\left(c_{i j}\right)$ and $K^{(0)}$ the distribution kernel of the positive contraction semigroup $S^{(0)}$ generated by $H_{0}$. The following conditions are equivalent:

I. There is a $\mu>0$ such that $C \geq \mu I$ almost everywhere.

II. There are $a, r>0$ such that for all $t \in\langle 0,1]$ one has

$$
K_{t}^{(0)}(x ; y) \geq a t^{-d / 2}
$$

for almost every $(x, y) \in \mathbf{R}^{d} \times \mathbf{R}^{d}$ with $|x-y| \leq r t^{1 / 2}$.

III. $K_{t}^{(0)}$ is a bounded function satisfying the Aronson Gaussian bounds (3).

The implication $\mathbb{I} \Rightarrow$ III follows by the Nash-Aronson estimates and obviously III $\Rightarrow$ II . Therefore the proof of the theorem is reduced to establishing that II $\Rightarrow$ I The proof is based on a variation of an argument of Carlen, Kusuoka and Stroock CKS which requires a different formulation of strong ellipticity.

The following proposition gives several related characterizations of strong ellipticity in terms of the forms $h, h_{0}$ and the corresponding operators. It is well known (see for example Folland [Fol], Theorem 7.17) that strong ellipticity is equivalent to a Gårding inequality, and this may be expressed in terms of either form.

Proposition 2. Let $H_{0}$ be the viscosity operator with coefficients $C=\left(c_{i j}\right)$. Moreover, let $h$ and $l$ be the forms given by (4) and (5) with common domain $\bigcap_{i=1}^{d} D\left(\partial_{i}\right)$, and let $h_{0}$ denote the form associated with $H_{0}$. The following conditions are equivalent:

I. The form $h$ is closed.

II. $h=h_{0}$.

III. There is a $\mu>0$ such that $C \geq \mu I$ almost everywhere.

IV. There is a $\mu>0$ such that $h \geq \mu l$.

V. There are $\mu>0$ and $\nu \geq 0$ such that $h \geq \mu l-\nu I$.

VI. There is a $\mu>0$ such that $H_{0} \geq \mu \Delta$ in the quadratic form sense.

VII. There are $\mu>0$ and $\nu \geq 0$ such that $H_{0} \geq \mu \Delta-\nu I$ in the quadratic form sense. 


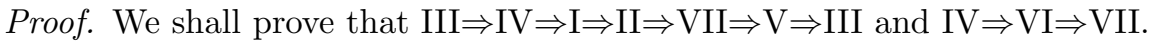

The implication $\Pi \cong$ in trivial. Since $h \leq\|C\| l$ and $l$ is closed, the implication IV $\Rightarrow$ is straightforward. The implication $\Pi \Rightarrow \Pi$ is trivial.

If $h=h_{0}$, then the vector space $D\left(\Delta^{1 / 2}\right)=D(h)=D\left(h_{0}\right)$ is a Banach space with respect to the norm $\varphi \mapsto\left(h_{0}(\varphi)+\|\varphi\|_{2}^{2}\right)^{1 / 2}$. But $D\left(\Delta^{1 / 2}\right)$ with the graph norm is also a Banach space. In addition both Banach spaces are continuously embedded in $L_{2}\left(\mathbf{R}^{d}\right)$. Hence there is a $c>0$ such that $\left\|\Delta^{1 / 2} \varphi\right\|_{2}^{2}+\|\varphi\|_{2}^{2} \leq c\left(h_{0}(\varphi)+\|\varphi\|_{2}^{2}\right)$ for all $\varphi \in D\left(\Delta^{1 / 2}\right)$ as a consequence of the closed graph theorem. Therefore one deduces VII

The implication $\nabla I I \Rightarrow \nabla$ is evident since $h_{0} \leq h$.

The implication $\mathrm{V} \Rightarrow$ III follows the proof of Theorem 7.17 in [Fol]. Let $\varphi \in$ $C_{c}^{\infty}\left(\mathbf{R}^{d}\right), k \in \mathbf{R}$ and $\xi \in \mathbf{R}^{d}$. Define $\varphi_{k} \in D(h) \cap D(l)$ by $\varphi_{k}(x)=e^{i k x \cdot \xi} \varphi(x)$. Then one calculates that

$$
\lim _{k \rightarrow \infty} k^{-2} h\left(\varphi_{k}\right)=\int_{\mathbf{R}^{d}} d x|\varphi(x)|^{2}(\xi, C(x) \xi) .
$$

Moreover,

$$
\lim _{k \rightarrow \infty} k^{-2}\left(\mu l\left(\varphi_{k}\right)-\nu\left\|\varphi_{k}\right\|_{2}^{2}\right)=\mu \int_{\mathbf{R}^{d}} d x|\varphi(x)|^{2}|\xi|^{2} .
$$

Since $h\left(\varphi_{k}\right) \geq \mu l\left(\varphi_{k}\right)-\nu\left\|\varphi_{k}\right\|_{2}^{2}$ one deduces that

$$
\int_{\mathbf{R}^{d}} d x|\varphi(x)|^{2}(\xi, C(x) \xi) \geq \mu \int_{\mathbf{R}^{d}} d x|\varphi(x)|^{2}|\xi|^{2} .
$$

Then one concludes that $C \geq \mu I$ almost everywhere. This proves the implication $\mathrm{V} \mapsto$ III

Next, if IV is valid, then $\mu l$ is a closed positive quadratic form with $\mu l \leq h$. Hence $\mu l \leq h_{0}$ and $\mathrm{VI}$ is valid. The implication $\mathrm{VI} \Rightarrow \mathrm{VII}$ is obvious.

As a final preliminary to the proof of the missing implication in Theorem 1 we need some information on Dirichlet forms [FOT], $\mathrm{BoH}$.

It is easy to verify that $h(|\varphi|) \leq h(\varphi)$ and $h(0 \vee \varphi \wedge \mathbb{1}) \leq h(\varphi)$ for all real valued $\varphi \in D(h)$. If $\varphi \in D\left(h_{0}\right)$ is real valued, then there are $\varphi_{1}, \varphi_{2}, \ldots \in D(h)$ such that $\lim \varphi_{n}=\varphi$ in $L_{2}$ and $h_{0}(\varphi)=\lim h\left(\varphi_{n}\right)$. Then $\lim \left|\varphi_{n}\right|=|\varphi|$ in $L_{2}$ and $\liminf h\left(\left|\varphi_{n}\right|\right) \leq \liminf h\left(\varphi_{n}\right)=h_{0}(\varphi)$. So $|\varphi| \in D\left(h_{0}\right)$ and $h_{0}(|\varphi|) \leq h_{0}(\varphi)$. Similarly, $0 \vee \varphi \wedge \mathbb{1} \in D\left(h_{0}\right)$ and $h_{0}(0 \vee \varphi \wedge \mathbb{1}) \leq h_{0}(\varphi)$. Therefore $h_{0}$ is a Dirichlet form and $S^{(0)}$ extends to a positive contraction semigroup on all the $L_{p}$-spaces, which we will also denote by $S^{(0)}$. It then follows from the positivity and contractivity that the semigroup $S^{(0)}$ satisfies

$$
0 \leq S_{t}^{(0)} \mathbb{1} \leq \mathbb{1}
$$

for all $t>0$ on $L_{\infty}\left(\mathbf{R}^{d}\right)$. (In fact one can prove that $S_{t}^{(0)} \mathbb{1}=\mathbb{1}$, but this is not straightforward (see [ERSZ, Proposition 3.6), and it is not necessary in the sequel.)

By the contractivity of $S^{(0)}$ and spectral theory one has

$$
h_{0}(\varphi) \geq t^{-1}\left(\varphi,\left(I-S_{t}^{(0)}\right) \varphi\right)
$$

for all $\varphi \in D\left(h_{0}\right)$ and $t>0$. But one deduces from (8) that

$$
\|\varphi\|_{2}^{2}=(\varphi, \varphi) \geq\left(S_{t}^{(0)} \mathbb{1},|\varphi|^{2}\right)=\left(|\varphi|^{2}, S_{t}^{(0)} \mathbb{1}\right)
$$


for all $t>0$, where $(\cdot, \cdot)$ denotes the duality between $L_{p}$ and $L_{q}$. Then it follows from self-adjointness of $S_{t}^{(0)}$ and (8) that

$$
h_{0}(\varphi) \geq(2 t)^{-1}\left(\left(S_{t}^{(0)} \mathbb{1},|\varphi|^{2}\right)+\left(|\varphi|^{2}, S_{t}^{(0)} \mathbb{1}\right)-\left(\varphi, S_{t}^{(0)} \varphi\right)-\left(S_{t}^{(0)} \varphi, \varphi\right)\right)
$$

for all $\varphi \in D\left(h_{0}\right)$ and $t>0$. This gives a related estimate in terms of the distribution kernel.

Let $\varphi, \chi \in C_{c}^{\infty}\left(\mathbf{R}^{d}\right) \subset D(h) \subseteq D\left(h_{0}\right)$. Suppose that $0 \leq \chi \leq 1$ and $\chi=1$ on the support of $\varphi$. Then since $S^{(0)}$ is positive, $S_{t}^{(0)} \chi \leq S_{t}^{(0)} \mathbb{1}$ and

$$
\begin{aligned}
h_{0}(\varphi) & \geq(2 t)^{-1}\left(\left(S_{t}^{(0)} \chi, \chi|\varphi|^{2}\right)+\left(\chi|\varphi|^{2}, S_{t}^{(0)} \chi\right)-\left(\chi \varphi, S_{t}^{(0)} \chi \varphi\right)-\left(S_{t}^{(0)} \chi \varphi, \chi \varphi\right)\right) \\
& =(2 t)^{-1} \int_{\mathbf{R}^{d} \times \mathbf{R}^{d}} d(x, y) K_{t}^{(0)}(x ; y) \chi(x) \chi(y)|\varphi(x)-\varphi(y)|^{2}
\end{aligned}
$$

for all $t>0$. This is the starting point of the Carlen-Kusuoka-Stroock argument to establish that $\Pi$ I in Theorem 1 .

End of proof of Theorem 1. Choose a smooth positive function $\rho$ with support in $\langle-r, r\rangle$ such that $\rho \leq 1$ and $\rho(x)=1$ for all $x \in \mathbf{R}^{d}$ with if $|x| \leq r / 2$. Then the previous estimate gives

$$
h_{0}(\varphi) \geq(2 t)^{-1} \int_{\mathbf{R}^{d} \times \mathbf{R}^{d}} d(x, y) K_{t}^{(0)}(x ; y) \rho\left(|x-y|^{2} t^{-1}\right) \chi(x) \chi(y)|\varphi(x)-\varphi(y)|^{2}
$$

for $\varphi \in C_{c}^{\infty}\left(\mathbf{R}^{d}\right), t>0$ and $\chi \in C_{c}^{\infty}\left(\mathbf{R}^{d}\right)$ such that $0 \leq \chi \leq 1$ and $\chi=1$ on the support of $\varphi$. Then it follows from Condition I that

$$
h_{0}(\varphi) \geq a(2 t)^{-1} \int_{\mathbf{R}^{d}} d x \int_{\mathbf{R}^{d}} d y t^{-d / 2} \rho\left(|x-y|^{2} t^{-1}\right) \chi(x) \chi(y)|\varphi(x)-\varphi(y)|^{2} .
$$

But the left-hand side is independent of the choice of $\chi$, so by the monotone convergence theorem

$$
h_{0}(\varphi) \geq a(2 t)^{-1} \int_{\mathbf{R}^{d}} d x \int_{\mathbf{R}^{d}} d y t^{-d / 2} \rho\left(|x-y|^{2} t^{-1}\right)|\varphi(x)-\varphi(y)|^{2}
$$

for all $\varphi \in C_{c}^{\infty}\left(\mathbf{R}^{d}\right)$ and $t \in\langle 0,1]$. Therefore if $\widehat{\varphi}$ denotes the Fourier transform of $\varphi$, then

$$
\begin{aligned}
h_{0}(\varphi) & \geq a t^{-1} \int_{\mathbf{R}^{d}} d x t^{-d / 2} \rho\left(|x|^{2} t^{-1}\right) \int_{\mathbf{R}^{d}} d \xi|\widehat{\varphi}(\xi)|^{2}(1-\cos \xi . x) \\
& =a t^{-1} \int_{\mathbf{R}^{d}} d x \rho\left(|x|^{2}\right) \int_{\mathbf{R}^{d}} d \xi|\widehat{\varphi}(\xi)|^{2}\left(1-\cos t^{1 / 2} \xi \cdot x\right) \\
& =2 a \int_{\mathbf{R}^{d}} d \xi|\widehat{\varphi}(\xi)|^{2} \int_{\mathbf{R}^{d}} d x \rho\left(|x|^{2}\right) t^{-1} \sin ^{2}\left(2^{-1} t^{1 / 2} \xi . x\right)
\end{aligned}
$$

for all $\varphi \in C_{c}^{\infty}\left(\mathbf{R}^{d}\right)$ and $t \in\langle 0,1]$. Thus in the limit $t \rightarrow 0$ one has

$$
h_{0}(\varphi) \geq 2^{-1} a \int_{\mathbf{R}^{d}} d \xi|\widehat{\varphi}(\xi)|^{2} \int_{\mathbf{R}^{d}} d x \rho\left(|x|^{2}\right)(\xi \cdot x)^{2}=\mu \int_{\mathbf{R}^{d}} d \xi|\widehat{\varphi}(\xi)|^{2}|\xi|^{2}=\mu l(\varphi)
$$

for all $\varphi \in C_{c}^{\infty}\left(\mathbf{R}^{d}\right)$ with $\mu>0$. Then since $h_{0} \leq h$, by the discussion preceding Theorem 1, one has $h(\varphi) \geq \mu l(\varphi)$ for all $\varphi \in C_{c}^{\infty}\left(\mathbf{R}^{d}\right)$. But the coefficients of $h$ are bounded and $C_{c}^{\infty}\left(\mathbf{R}^{d}\right)$ is a core for $W^{1,2}\left(\mathbf{R}^{d}\right)=D(l)=D(h)$. So $h(\varphi) \geq \mu l(\varphi)$ for all $\varphi \in D(l)$. Thus Condition IV] in Proposition 2 is satisfied. But this is equivalent 
to Condition $\amalg$ of the proposition which is just a repetition of the strong ellipticity hypothesis, Condition \in Theorem 1 .

Although much work in recent years has been devoted to the derivation of Gaussian upper bounds on semigroup kernels, Theorem 1 demonstrates that Gaussian lower bounds are in fact the important feature in understanding the general behaviour of the kernels. The local small time lower bounds in Condition II of the theorem encapsulate all the information contained in the Aronson upper and lower bounds. The lower bounds reflect the correct small $t$ behaviour, and this is enough to derive the behaviour, of the semigroup and its kernel for all $t$.

It is also interesting to note that in quite general circumstances (see, for example, $\mathrm{Cou}$ ) the Gaussian upper bounds suffice to prove that Gaussian lower bounds are equivalent to Hölder continuity of the kernel. In particular each of the equivalent conditions of the theorem implies that the semigroup kernel is Hölder continuous.

It is also possible to extend the theorem to the setting of subelliptic operators on Lie groups. Let $a_{1}, \ldots, a_{d}$ be a vector space basis for the Lie algebra $\mathfrak{g}$ of a Lie group $G$. For all $i \in\{1, \ldots, d\}$ let $A_{i}$ be the infinitesimal generator of the one parameter group $t \mapsto L\left(\exp \left(-t a_{i}\right)\right)$, where $L$ is the left regular representation in $L_{2}(G)$. For all $i, j \in\{1, \ldots, d\}$ let $c_{i j} \in L_{\infty}(G)$ and suppose that the matrix $\left(c_{i j}\right)$ is real, symmetric and positive-definite almost everywhere. One can define as above a viscosity operator $H_{0}$ corresponding to the formal expression $-\sum_{i, j=1}^{d} A_{i} c_{i j} A_{j}$.

Next let $d^{\prime} \leq d$ and suppose that $a_{1}, \ldots, a_{d^{\prime}}$ generate the Lie algebra $\mathfrak{g}$. Associated to $a_{1}, \ldots, a_{d^{\prime}}$ one can define a modulus $|\cdot|^{\prime}$ on $G$ and a local dimension $D^{\prime} \in \mathbf{N}$, i.e., $\operatorname{Vol}\left\{g \in G:|g|^{\prime}<\rho\right\} \asymp \rho^{D^{\prime}}$ for $\rho \in\langle 0,1]$. Then one has the following theorem.

Theorem 3. Let $H_{0}$ be the viscosity operator with coefficients $\left(c_{i j}\right)$ and let $K^{(0)}$ be the distribution kernel of the positive contraction semigroup $S^{(0)}$ generated by $H_{0}$. The following conditions are equivalent:

I. There is a $\mu>0$ such that

$$
\left(\varphi, H_{0} \varphi\right) \geq \mu \sum_{i=1}^{d^{\prime}}\left\|A_{i} \varphi\right\|_{2}^{2}
$$

for all $\varphi \in \bigcap_{i=1}^{d^{\prime}} D\left(A_{i}\right)$.

II. There are $a, r>0$ such that for all $t \in\langle 0,1]$ one has

$$
K_{t}^{(0)}(g ; h) \geq a t^{-D^{\prime} / 2}
$$

for almost every $(g, h) \in G \times G$ with $\left|g h^{-1}\right|^{\prime} \leq r t^{1 / 2}$.

III. There are $a, a^{\prime}, b, b^{\prime}, \omega, \omega^{\prime}>0$ such that

$$
a^{\prime} t^{-D^{\prime} / 2} e^{-\omega^{\prime} t} e^{-b^{\prime}\left(\left|g h^{-1}\right|^{\prime}\right)^{2} t^{-1}} \leq K_{t}^{(0)}(g ; h) \leq a t^{-D^{\prime} / 2} e^{\omega t} e^{-b\left(\left|g h^{-1}\right|^{\prime}\right)^{2} t^{-1}}
$$

for all $t>0$ and $g, h \in G$.

The implication $\llbracket \Leftrightarrow \Pi$ in in ElR2, the implication $\amalg I I \Pi$ is trivial and the implication $\Pi \rrbracket$ is as in the proof of Theorem 1 . But instead of the scaling of $\rho$ used in the above proof one has to use the maps $\gamma_{t}$ as in [ElR1], Section 3. We omit the technical details.

Finally the situation is quite different for second-order real divergence form operators which are degenerate [ERSZ. Then the kernel is positive but not necessarily 
strictly positive even if the operator is subelliptic. One may construct examples for which the kernel vanishes on the loci of degeneracy. In particular one cannot expect any type of Gaussian lower bound. Nevertheless subellipticity and a condition of uniform strict positivity suffice to deduce Gaussian upper bounds which incorporate the correct large $t$ behaviour (see ERSZ for details).

\section{REFERENCES}

[Aro] Aronson, D.G., Bounds for the fundamental solution of a parabolic equation. Bull. Amer. Math. Soc. 73 (1967), 890-896. MR0217444 (36:534)

[BoH] Bouleau, N. and Hirsch, F., Dirichlet forms and analysis on Wiener space, vol. 14 of de Gruyter Studies in Mathematics. Walter de Gruyter \& Co., Berlin, 1991. MR1133391 (93e:60107)

[CKS] Carlen, E.A., Kusuoka, S. and Stroock, D.W., Upper bounds for symmetric Markov transition functions. Ann. Inst. Henri Poincaré 23 (1987), 245-287. MR0898496 (88i:35066)

[Cou] Coulhon, T., Off-diagonal heat kernel lower bounds without Poincaré. J. London Math. Soc. 68 (2003), 795-816. MR2010012 (2004m:58043)

[Dal] Dal Maso, G., An introduction to $\Gamma$-convergence, vol. 8 of Progress in Nonlinear Differential Equations and their Applications. Birkhäuser Boston, Inc., Boston, MA, 1993. MR.1201152 (94a:49001)

[Dav] Davies, E.B., Heat kernels and spectral theory. Cambridge Tracts in Mathematics 92. Cambridge University Press, Cambridge, 1989. MR0990239 (90e:35123)

[DeG] De Grorgi, E., Sulla differenziabilità e l'analiticità delle estremali degli integrali multipli regolari. Mem. Accad. Sci. Torino cl. Sci. Fis. Mat. Nat. 3 (1957), 25-43. MR0093649 (20:172)

[DER] Dungey, N., ter Elst, A.F.M. and Robinson, D.W., Analysis on Lie groups with polynomial growth, vol. 214 of Progress in Mathematics. Birkhäuser Boston, Inc., Boston, 2003. MR2000440 (2004i:22010)

[EkT] Ekeland, I. and Temam, R., Convex analysis and variational problems. North-Holland Publishing Co., Amsterdam, 1976. MR0463994 (57:3931b)

[ElR1] TER Elst, A.F.M. and Robinson, D.W., Weighted subcoercive operators on Lie groups. J. Funct. Anal. 157 (1998), 88-163. MR1637929 (99h:47055)

[EIR2] Second-order subelliptic operators on Lie groups II: real measurable principal coefficients. In Balakrishnan, A. V., ed., Proceedings for the First International Conference of Semigroups of Operators: Theory and Applications, Newport Beach, California, vol. 42 of Progress in Nonlinear Differential Equations and their Applications. Birkhäuser Verlag, Basel, 2000, 103-124. MR 1788873(2001i:35053)

[ERSZ] ter Elst, A.F.M., Robinson, D.W., Sikora, A. and Zhu, Y., Second-order operators with degenerate coefficients, 2004.

[Fol] Folland, G.B., Introduction to partial differential equations. Mathematical Notes 17. Princeton University Press, Princeton, 1976. MR.0599578 (58:29031)

[FOT] Funushima, M., Oshima, Y. and Takeda, M., Dirichlet forms and symmetric Markov processes, vol. 19 of de Gruyter Studies in Mathematics. Walter de Gruyter \& Co., Berlin, 1994. MR1303354 (96f:60126)

[Gri] Grigor'yan, A., Estimates of heat kernels on Riemannian manifolds. In Spectral theory and geometry (Edinburgh, 1998), vol. 273 of London Math. Soc. Lecture Note Ser., 140-225. Cambridge Univ. Press, Cambridge, 1999. MR1736868 (2001b:58040)

[Kat] Kato, T., Perturbation theory for linear operators. Corrected printing of the second edition, Grundlehren der mathematischen Wissenschaften 132. Springer-Verlag, Berlin, New York, 1980. MR0407617(53:11389)

[Nas] NASh, J., Continuity of solutions of parabolic and elliptic equations. Amer. J. Math. 80 (1958), 931-954. MR0100158 (20:6592)

[Sim1] Simon, B., Lower semicontinuity of positive quadratic forms. Proc. Roy. Soc. Edinburgh Sect. A 79 (1977), 267-273. MR0512713 (58:23685)

[Sim2] A canonical decomposition for quadratic forms with applications to monotone convergence theorems. J. Funct. Anal. 28 (1978), 377-385. MR0500266 (58:17937) 
[Str1] Stroock, D.W., Diffusion semigroups corresponding to uniformly elliptic divergence form operators. In AzÉma, J., Meyer, P.A. and Yor, M., eds., Séminaire de probabilités XXII, Lecture Notes in Mathematics 1321. Springer-Verlag, Berlin, 1988, 316-347. MR0960535 (90b:35071)

[Str2] , Estimates for the heat kernel of second order elliptic operators. In Nonlinear partial differential equations and their applications. Collège de France Seminar, Vol. XII (Paris, 1991-1993) 226-235, vol. 302 of Pitman Res. Notes Math. Ser., Longman Sci. Tech., Harlow, 1994. MR 1291854 (95h:35054)

Centre for Mathematics and its Applications, Mathematical Sciences Institute, Australian National University, Canberra, ACT 0200, Australia

Current address: Department of Mathematics and Computing Science, Eindhoven University of Technology, P.O. Box 513, 5600 MB Eindhoven, The Netherlands

E-mail address: terelst@win.tue.nl

Centre for Mathematics and its Applications, Mathematical Sciences Institute, Australian National University, Canberra, ACT 0200, Australia

E-mail address: Derek.Robinson@anu.edu.au

Centre for Mathematics and its Applications, Mathematical Sciences Institute, Australian National University, Canberra, ACT 0200, Australia

Current address: Department of Mathematics, Nantong University, Nantong, 226007, Jiangsu Province, People's Republic of China

E-mail address: zhuyueping@ntu.edu.cn 